\title{
Políticas municipales de fomento de exportaciones locales en Argentina: implicancias de su condición de territorios periféricos
}

\author{
GERMÁn DABAT*
}

\begin{abstract}
The promotion of exports of local producers by the Argentinean local municipal governments in the past decade has been influenced by two diverging theories of the local productive devel opment: one aims to achieve devel opment through the dynamics provided by the market, whereas the other one aspires to the construction of social development. In order for the process to gather momentum, it is necessary to establish a positive feedback loop between exportingand thelocal productive devel opment, which requiresa strengthening of themarket mechanisms to counteract theintenseweaknesses shown in Argentina, and at the sameto reinforcetheincipient strengths. To solvethis problem, weelucidate about different ways in which the available assets are valued for the development in the centreand in the periphery, emphasising on the different resources available in each place. We classify these assets into those that havethe capacity to self-reproduce, to self-value or valuethe rest of the availableassets and those that have a static character.
\end{abstract}

Keywords international trade, local development, policies.

\begin{abstract}
Resumen
El fomento de exportaciones de los productores locales por gobiernos municipales argentinos en la década pasada se ha regido por dos influencias teóricas divergentes sobre el desarrollo productivo local: una pretende el desarrollo mediante el dinamismo que provee el mercado y la otra aspira a la construcción social del desarrollo. Para que cobre impulso el proceso esperado por los gestores de estas políticas, debe conformarse un círculo virtuoso entre la exportación y el desarrollo productivo local, lo que requiere reforzar los mecanismos del mercado que contrarreste las fuertes debilidades mostradas en Argentina y afiance sus incipientes fortal ezas. Para solucionar este problema, aquí se propone la reflexión en torno a las diversas maneras en que se valoran los activos disponibles para el desarrollo en el centro y en la periferia, con énfasis en la diferente dotación de activos disponibles en cada lugar, clasificando entre aquellos que tienen capacidad de autorreproducción, de autovaloración o de valoración del resto de los activos disponibles, y aquellos que tienen un carácter estático.
\end{abstract}

Palabras clave: comercio internacional, desarrollo local, política/s.

*Universidad Nacional de Quilmes. Correo-e: gdabat@unq.edu.ar. 


\section{Introducción ${ }^{1}$}

En la década pasada, un número considerable de gobiernos municipales argentinos se hizo cargo de la realización de actividades de fomento de exportaciones de los productores locales. Esto constituye un fenómeno nuevo en la Argentina y muy poco usual en otros países. Esas actividades anteriormente sólo eran realizadas por instancias gubernamentales nacionales y provinciales. Como lo demuestra A. Villar (2004), esa descentralización acompañó a un proceso más amplio de derivación hacia los municipios de otras actividades productivas, en el marco de un intento de fomentar procesos de desarrollo local endógeno ${ }^{2}$ (DLE). En este artículo se mostrará que esas iniciativas comerciales pretenden realizar un aporte positivo a los procesos de desarrollo local respectivos, aunque hasta el momento han mostrado resultados muy modestos. ${ }^{3}$

El desafío planteado aquí consiste en analizar la capacidad de la exportación de bienes para promover procesos de evolución productiva territorial. M ás particularmente, se pretende anali-

\footnotetext{
${ }^{1}$ El presente artículo fue elaborado en el marco del proyecto Experiencias de desarrollo local y comercio exterior, de la Universidad $\mathrm{N}$ acional de Quilmes, y refleja en forma sintética el trabajo de tesis doctoral Las políticas municipales de competitividad y comercio exterior en Argentina como instrumento del desarrollo local endógeno, que está en proceso de discusión del borrador final en la Universidad Autónoma de Zacatecas.

${ }^{2}$ La teoría del desarrollo endógeno parte de la idea de que el desarrollo económico es consecuencia de la aplicación del conocimiento en los procesos productivos y la utilización de las economías externas que se generan en los sistemas productivos y en las ciudades. Pone énfasis en que en ese proceso hay rendimientos crecientes $y$, por lo tanto, crecimiento económico, gracias a la utilización del potencial y el excedente generado localmente y a la eventual atracción, de recursos externos (Vázquez Barquero, 1999: 20).

${ }^{3}$ La investigación de campo realizada en los dos proyectos mencionados anteriormente comprendió la aplicación de una encuesta genérica a 119 empresarios del municipio de Florencio Varela, nueve de los cuales fueron objeto de entrevistas en profundidad para verificar si a partir de sus exportaciones tuvieron aprendizaje productivo y si consideraban que habían realizado algún tipo de transmisión de ese conocimiento en el entorno local, en cuyo caso se explican los mecanismos de transmisión social del mismo. Por otra parte, se realizó una consulta por vía de correo electrónico a todos los municipios del país para averiguar en cuáles de ellos hay oficinas municipales de comercio exterior (véase anexo). Por último, se realizaron entrevistas en profundidad con funcionarios y ex funcionarios de ocho municipios de la Provincia de Buenos Aires que tienen distintas características estructurales entre sí para cubrir los diferentes perfiles de gobiernos municipales que realizan actividades de comercio exterior; con ellos se indagó sobre los motivos políticos que los condujeron a realizarlas. Los funcionarios entrevistados corresponden a los municipios de Lomas de Zamora, Vicente López, Q uilmes, Florencio Varela, M ar del Plata, Junín, y Olavarría y Rauch, lo que cumple el amplio espectro de estructuras municipales que existen en la provincia de Buenos Aires.
} 
zar la potencialidad que existe en Argentina para que las exportaciones catalicen el desarrollo del territorio y la función que cumplen los gobiernos municipales para favorecer ese proceso. De acuerdo con el enfoque asumido aquí, esto nos lleva al análisis de las diferencias existentes entre las potencialidades de incidencia de la exportación de bienes sobre el DLE en ciudades periféricas situadas en países periféricos (CPPP), por un lado, y en ciudades periféricas situadas en países centrales (CPPC), poniendo énfasis en problemas de reproducción endógena, entre los que destacan los vinculados con el aprendizaje productivo.

El tema está tratado desde la perspectiva de las teorías del comercio internacional que se apoyan en el análisis de características estructurales territoriales, ya sean naturales o construidas socialmente, más que en la habilidad empresarial para hacer negocios. En ese contexto, la estructura económica local está definida por el tipo de sectores que operan en ella (industrial, semi-industrial, agropecuaria, minera 0 de no transables, por ejemplo), su dotación de factores, su tamaño, la tradición exportadora de los productores locales, la composición de su producción potencialmente exportable (commodities o diferenciados), etc., $y$, muy especialmente en las últimas décadas, por factores que se autorreproducen y autovaloran, como el conocimiento y la organización de la producción, dentro lo cual un ejemplo significativo es el trabajo de Porter (1991).

Para ello se toman los siguientes antecedentes teóricos:

- Finquielevich (2001) aporta la distinción entre ciudades periféricas y ciudades centrales. En el marco del proceso de aumento de las relaciones internacionales que caracteriza a la globalización se va conformando una estructura reticular en la que se diferencian crecientemente ambos tipo de ciudades. Las ciudades centrales son las que se vinculan activamente con el resto del mundo, y las periféricas son las que tienden a quedar cada vez más aisladas.

- Una significativa cantidad de investigaciones empíricas de las dos últimas décadas destaca la trascendencia de la relación existente entre la exportación de bienes y el aprendizaje de las firmas exportadoras. Los resultados de muchos de esos análisis están reflejados por una serie creciente de autores que discuten la investigación pionera de Johanson y Wiedersheim-Paul (1975) y Johanson y Valhne (1977), entre los que se encuentran Lee y Brasch (1978); Johan- 
son y Vahlne (1990), Brown y Cook (1990), C avusgil, (1998) y Ellis (2000).

- O tros autores, como Krugman (1992), tratan extensamente la relación entre comercio internacional y geografía económica, como un mecanismo de retroalimentación en el que la estructura territorial posibilita y potencia el comercio y éste fortalece la competitividad internacional del territorio.

- Kosacoff y López (1998) destacan la reemergencia de pequeñas y medianas empresas (pymes) como protagonistas de la reestructuración económica en países desarrollados, cuando están arraigadas a territorios competitivos internacionalmente.

- Saba (1997) analiza la estrecha relación entre exportación de bienes diferenciados a mercados de alto poder adquisitivo de la Unión Europea y desarrollo de los distritos industriales.

- Bianchi (1997) sostiene que el éxito de las empresas en Europa se basa en aglomeraciones de firmas con diversas especialidades que trabajan en forma complementaria y que se convierten en el sujeto que protagoniza la competencia.

- Becattini y Rullani (1996), a partir de sus estudios empíricos en el norte de Italia, sostienen que el territorio, además de ser un espacio de realización de actividades económicas, es un recurso específico y un actor principal del desarrollo económico.

- Vázquez Barquero (1999) aporta la teoría del DLE, en la que se observa la importancia de la acción social para construir el desarrollo y la inserción internacional. En ese sentido, sostiene que las estrategias de construcción del entorno competitivo deben "incluir tanto al comercio internacional como a la inversión extranjera, ya que ambos pueden influir en el logro de mayores niveles de productividad y competitividad". Puede verse en ella cierta influencia favorable de la exportación de bienes diferenciados en el desarrollo de ciudades periféricas situadas en países centrales 0 en el desarrollo de algunos sectores o clusters productivos pertenecientes a ciudades centrales situadas en aquellos países. Esa teoría fue enunciada después de observarse tendencias en el proceso de desarrollo de ciertas ciudades periféricas situadas en países centrales. En general se observaron procesos del norte de España y del 
norte de Italia. Es decir, en las regiones más ricas de cada uno de esos dos países.

- Dabat (2003), D abat y Le Clech (2004), Leal (2004) y Segreto (2004) señalan la desarticulación existente entre las políticas municipales de comercio exterior (PMCE) y las estrategias de desarrollo local en Argentina.

Teniendo en cuenta estos antecedentes teóricos, en este artículo se plantea que la relación exportación de bienes diferenciados-desarrollo local en las cppp tiene ciertas especificidades no consideradas en la teoría del DLE elaborada en Europa. Se fija la atención en este punto para discutir el rol que las $\mathrm{PM} \mathrm{CE}^{4}$ pueden cumplir como catalizadores de procesos de evolución productiva territorial. Por eso, el objetivo de este trabajo es señalar las dificultades existentes para que las exportaciones fomentadas cumplan dicho rol cuando se apoyan sólo en los mecanismos de mercado -sin respaldo de políticas focalizadas que faciliten el proceso-, y la función que potencialmente puede cumplir la exportación de bienes para incidir sobre estilos, ritmos y resultados en los procesos de desarrollo productivo local en Argentina, considerando que su dotación relativa de activos (o recursos) dinámi$\cos ^{5}$ disponibles para el desarrollo es menor que la disponible en las localidades en europeas en las que se inspiró aquella teoría.

Para cumplir con ese objetivo se dividió el artículo en cuatro partes. En la primera se señalan las enseñanzas que dejan las experiencias exitosas de desarrollo local o microrregional de los países centrales para los países periféricos, lo que luego se tomará como antecedente para señalar la potencialidad de laS PM CE como factor de la aceleración de procesos de desarrollo productivo local. En la segunda parte se definen las PM CE aplicadas en Argentina desde la década pasada y se describen los objetivos potenciales y reales de esas políticas, subrayándose especialmente sus vínculos con la promoción de procesos de creación y absorción social de conocimiento productivo, característica prin-

${ }^{4}$ En otro trabajo (D abat, 2005) se concluyó que la observación de esas diferencias es útil para entender el sentido político de las actividades de fomento de exportaciones que realizan algunos gobiernos municipales argentinos, a las que se calificó como PM CE. N ótese que las PM CE son implícitas; no están definidas como tales por los municipios que las llevan a cabo. La caracterización como "políticas" para el conjunto de actividades de fomento de exportaciones realizadas por los gobiernos municipales surge de la interpretación de sentido a partir de la observación de ciertas regularidades y de las declaraciones realizadas por los funcionarios municipales entrevistados (véase la nota 3).

${ }^{5}$ El carácter dinámico de ciertos activos está expuesto en el cuarto apartado de este artículo. 
cipal de las experiencias de DLE observadas en Europa. En la tercera parte se exponen las expectativas sobre que laS PMCE podrían incidir favorablemente sobre el valor estratégico ${ }^{6}$ de los activos locales disponibles para el desarrollo si se adecuaran a las condiciones precarias reinantes en la Argentina, creando un círculo virtuoso entre la exportación y el desarrollo productivo local. Por último, en la cuarta parte, teniéndose en cuenta las experiencias internacionales exitosas (1) y las expectativas cifradas en las PM CE (3), se realiza un breve ensayo en el que se sintetiza la lógica del proceso de valoración estratégica de los activos disponibles para el desarrollo. La propuesta realizada en ese último apartado gira en torno a la valoración estratégica de activos sociales y privados disponibles para el desarrollo, cuando la estrategia municipal requiere el uso intensivo de conocimiento productivo, de organización de la producción, de marcas locales y demás activos con capacidad potencial de autorreproducción. A esos efectos, el cuarto punto ofrece una reflexión sobre las formas diferentes en las que se valoran los activos disponibles para el desarrollo en el centro y en la periferia, como consecuencia de su diferente dotación de activos disponibles.

\section{Enseñanzas de las experiencias exitosas internacionales desde la perspectiva del desarrollo local endógeno}

Las últimas décadas se caracterizaron por el aumento de la importancia de los procesos de descentralización de actividades y la competencia nacional e internacional entre territorios subnacionales. Ese nuevo fenómeno explica el surgimiento de la teoría del DLE, que parte de la observación de cambios estructurales producidos en diversos países desarrollados. Esa teoría asocia los cambios tecnológicos y organizacionales con la construcción endógena del desarrollo económico local, lo que se refleja en numerosos trabajos como los de Vázquez Barquero (1993, 1999 y 2005), Alburquerque (1995 y 2004) y Silva Lira (2003).

\footnotetext{
${ }^{6} \mathrm{C}$ abe destacar la diferencia existente entre el concepto de valor estratégico de los activos y el de desarrollo endógeno local en sí. El primero se refiere al valor que tienen los activos sociales y privados en función de la estrategia de desarrollo endógeno local. Por su parte, el segundo concepto se refiere a una construcción social que requiere disponer de activos valorados para su ejecución. Por lo tanto, la valoración de un activo desde el punto de vista estratégico; es decir, en el marco de una estrategia de desarrollo, puede no coincidir con su precio en el mercado o con su rentabilidad esperada desde el punto de vista privado.
} 
Ese avance tecnológico trajo consigo una serie de modificaciones en la organización del trabajo intra e inter firmas. Uno de los cambios más importantes que se verificaron en ese proceso es el de los ámbitos en los que se explotan las economías de escala y en los que reside la flexibilidad. En el esquema fordista preexistente había grandes economías de escala en la producción de bienes. En cambio, en la nueva estructura flexible se redujeron esas economías en la producción de bienes debido a la disminución del tamaño de la planta tipo en muchos sectores industriales, pero aumentaron dichas economías en la producción de tecnología ${ }^{7}$ y en las estructuras productivas intensivas en tecnologías de la información y la comunicación. ${ }^{8}$ Como lo describen Kosacoff y López (1998), ese fenómeno es especialmente importante en los países desarrollados, donde el tamaño medio de las plantas industriales está reduciéndose en procura de mayor flexibilidad. Es decir, se prefiere la rápida adaptación a situaciones cambiantes cuantitativa o cualitativamente, en lugar de la fabricación en serie de productos estandarizados. La nueva demanda de bienes de capital llevó a los fabricantes de éstos a reducir el tamaño de sus productos y, por lo tanto, a hacerla más adecuada a las necesidades de las pymes en el nuevo contexto, devolviéndoles funcionalidad y convirtiéndolas un soporte fundamental para el dinamismo del sistema. La reducción de la escala de producción óptima en la producción de bienes implica una descentralización de actividades, incluso hacia países periféricos. A su vez, el aumento de la escala en la producción de tecnologías, diseño y gestión de la marca requiere de un vasto mercado para el cual producir.

Por su parte, el efecto de la difusión internacional de las nuevas tecnologías de la información y la comunicación, sumado al encarecimiento de los costos del comercio internacional evidenciado desde comienzos de la década de 1980 (Alburquerque, 1995), alteró los costos relativos de la producción descentralizada respec-

${ }^{7}$ Las actividades tecnológicas requieren crecientes gastos fijos cuanto más sofisticada es la actividad innovativa de la firma o institución y, al menos para la teoría económica tradicional, tienen un costo marginal igual a cero (Dosi, Pavitt y Soete, 1990; Katz, 1976).

${ }^{8}$ La economía de la información creó los cimientos del nuevo paradigma tecnoproductivo que desplazó al anterior con base en ventajas propias de tecnologías más dinámicas y flexibles. Los bits desplazan a los átomos del centro de dinamismo del sistema. Los bits pueden reproducirse sin costo y distribuirse por todo el mundo a la velocidad de la luz y nunca se deterioran. Los bienes materiales son caros de producir y de transportar y se deterioran inevitablemente (Varian, 1998). 
to a la de gran escala para exportación, en favor de la primera. Se creó así un escenario propicio para trasladar menos bienes y más información (Castells, 2001). Por lo tanto, se crearon condiciones para abaratar la producción, acelerar la rotación del capital, discriminar precios de acuerdo con las características de la demanda y diferenciar los bienes producidos. Así se potenciaron las nuevas características técnicas de los bienes de capital con la infraestructura de comunicación, lo que favorece el aumento de la productividad del trabajo. En ese nuevo contexto tecnológico, el comercio internacional, como herramienta de desarrollo de ciudades periféricas, puede pasar a ser más importante por el aprendizaje productivo que pueden realizar las firmas y clusters exportadores que por la explotación de economías de escala.

N o obstante, la copia de modelos exitosos no es simple, como lo demuestra la dificultad para difundir territorialmente la eficiencia productiva que se observa en los países en los que se llevan a cabo esas experiencias. Probablemente uno de los ejemplos más citados sobre cómo obtener éxito en el nuevo contexto sea el de los distritos italianos. En Italia está ocurriendo un fenómeno de polarización entre atraso y desarrollo. M ientras en los distritos industriales cercanos a Venecia, Florencia y Bolonia, e incluso en parte del centro del país, hay pleno empleo y aumenta la productividad del trabajo y los niveles de vida, en otras regiones aumenta la desocupación y están estancadas tecnológicamente (Saba, 1997: 23).9

Según Saba (1997), los elementos clave de la competitividad del norte de Italia son:

- La productividad del trabajo en el sistema industrial. En ese tipo de economías, las innovaciones tecnológicas surgen de la unión de muchas empresas extremadamente especializadas. La articulación de sus esfuerzos de especialización para el crecimiento continuo de la productividad les permite hacer frente al mercado internacional. Dicha competitividad internacional se alcanzó por la extrema especialización de la maquinaria y herramienta, lo que requiere de un nivel cultural e industrial muy alto. La evolución continua se apoya en el alto conocimiento de trabaja-

${ }^{9}$ La teoría del desarrollo local endógeno interpreta que la capacidad de algunos territorios para desarrollarse se debe a la cultura productiva arraigada en la sociedad durante mucho tiempo. Por lo tanto, serían razones sociales las que explicarían el éxito de las firmas individuales. 
dores calificados en mecánica e informática, por lo que dominan la maquinaria con la que trabajan. El resultado de esa experiencia es que Italia se convirtió en el tercer país, después de Alemania y Japón, en la producción y exportación de maquinaria y herramienta. Esa posición, y el desplazamiento de Estados Unidos, fue lograda gracias a la especialización en maquinaria de pequeñas dimensiones. El alto crecimiento del Norte italiano se debe a la división internacional del trabajo: Italia le compra la maquinaria grande a Alemania, y Alemania es la principal compradora de maquinaria pequeña italiana, incluso por sobre el mercado interno italiano. Por lo tanto, el fenómeno italiano se apoya en su articulación con el resto de Europa.

- La integración interna, por lo que se construyó una economía flexible basada en la especialización de las firmas. La maquinaria y herramienta de pequeña escala que se utiliza en cada distrito se produce en el propio distrito. Gracias a la cercanía territorial, cuando hay un nuevo proceso de producción o un nuevo producto en fase experimental la pequeña empresa productora de bienes finales puede mantener un diálogo continuo entre los productores de maquinaria y herramienta. Ese diálogo posibilitó un doble progreso: de los productores de maquinaria y herramienta en el perfeccionamiento continuo de sus productos, y en los productores de bienes finales en la eficiencia con la que los utilizaban.

- La flexibilidad. Dado que el modelo italiano se apoya en su articulación con el resto de Europa, la inestabilidad permanente que caracteriza al mercado internacional exige una alta flexibilidad de la estructura industrial para amortiguar los impactos. La flexibilidad permitió la adaptación en forma eficiente y rápida frente a shocks en las finanzas internacionales, al cambio tecnológico y a la variación de los precios internacionales.

- La calidad. En vez de basar su especialización en una estrategia de abaratamiento de costos por producción en serie, los distritos industriales italianos procuran la evolución cualitativa de sus productos. Se especializaron en producir y vender productos diferenciados para mercados de alto poder adquisitivo, compitiendo en el mercado internacional más por calidad que por precio. Esa decisión estuvo inspirada en la observación de que en el mercado internacional la 
cantidad física comercializada se reduce continuamente, pero la cantidad en valor aumenta continuamente.

Entonces, surge la pregunta: ¿si en el sur de Italia el poderoso gobierno nacional y la Unión Europea llevan tres décadas de fracasos en sus intentos por acortar la brecha de productividad entre el Sur y el N orte italiano, por qué suponer que es posible el desarrollo de las cppp siguiendo la receta de los distritos industriales italianos? Parece evidente que ninguno de los recursos utilizados en el Norte italiano da garantías de éxito.

No obstante, los cambios tecnológicos y organizacionales operados en el centro inciden favorablemente en la industrialización de la periferia por dos razones que actúan en forma paralela y son complementarias. La primera razón consiste en la creación de la necesidad de modernización. Esto es, la presión que ejercen el aumento de la productividad del trabajo, la reducción de precios de los bienes industrializados en el mercado mundial, y el riesgo de desplazamiento de los mercados internacionales por países periféricos que adquieren tecnologías de producción más avanzadas, entre otros. La segunda razón es la posibilidad de aprender que ofrece el nuevo panorama, ya sea gracias a la importación y exportación de bienes industriales, de la inversión extranjera directa, de la cooperación internacional, de franquicias u otras formas de asociación con empresas extranjeras de tecnología avanzada, de las actividades de investigación y desarrollo que estudian productos o procesos situados en la frontera del conocimiento mundial, etcétera.

El hecho de que esas dos razones favorezcan las condiciones de industrializar territorios periféricos, no implica que faciliten la realización de procesos de desarrollo autónomo. Por el contrario, esas condiciones suelen generar procesos de modernización inducida y dependiente. Para que esa evolución en las prácticas productivas redunde en desarrollo local endógeno, debe cumplirse como condición necesaria el ejercicio del protagonismo en la modernización por parte de los actores locales. En otras palabras, las nuevas condiciones tecnológicas internacionales obligan a los actores locales a redoblar esfuerzos siguiendo el ritmo de cambios impuesto por un mundo liderado por ciudades que se desarrollan mediante una combinación de valoración de activos sociales locales y acción social para el desarrollo endógeno. 


\section{O bjetivos desarrollistas de las PM CE}

En ese contexto internacional, algunos gobiernos municipales argentinos buscaron los medios para apoyar la inserción de empresas locales en el mercado internacional. Para ello realizan actividades relativamente simples, aunque en varios municipios éstas tienen una complejidad creciente a lo largo de su corta vida. En los casos relevados, esas actividades no son realizadas en el marco de una política cuyos objetivos son expuestos explícitamente. Las entrevistas brindadas por los funcionarios municipales de comercio exterior -mencionadas en la nota 3- demuestran que Ias PM CE están implícitas en las actividades realizadas, aunque el sentido político de las mismas puede ser interpretado por medio de la concepción política desde la que se generan expectativas de que las exportaciones promovidas incidan sobre los procesos de desarrollo productivo local.

Es decir, esas políticas se fueron configurando, de hecho, en forma tácita, a medida que el gobierno nacional y los gobiernos provinciales descentralizaron actividades tendentes a facilitar o promover las exportaciones de los productores locales y que los propios gobiernos municipales fueron respondiendo a demandas puntuales de esos productores con el fin de compensar los problemas de competitividad creados por la sobrevaluación cambiaria de la década pasada (D abat, 2005). Por lo tanto, tampoco fueron enunciados en forma sistemática sus objetivos. N o obstante, la aspiración de realizar un aporte al desarrollo productivo local mediante las actividades proexportadoras del gobierno municipal fue enfatizada por todos los funcionarios municipales entrevistados. Esa aspiración y los mecanismos previstos para lograrla tienen implícitas ciertas concepciones sobre el significado del desarrollo y la forma como se lo construye. ${ }^{10}$

Por otra parte, la experiencia internacional muestra que si bien el comercio internacional no puede sustituir a la construcción social del desarrollo, puede ser una herramienta útil para fortalecerlo (Saba, 1997; D abat, 2005). Entonces, cabe la pregunta: ¿es posible la utilización de las PM CE para fortalecer experiencias de DLE en Argentina? Si bien la respuesta no es simple ni lineal, surge preocupación al verificar, por medio de la investiga-

${ }^{10}$ La metodología de investigación utilizada para indagar sobre el significado que se le asigna a los procesos de desarrollo local catalizados por exportaciones está ampliamente descrita en la tesis doctoral citada al comienzo del presente artículo. 
ción empírica (D abat, 2003; D abat y Le Clech, 2004; Leal, 2004; Segreto 2004), que existe cierta tendencia en este país a la realización de actividades municipales orientadas al comercio internacional en las que los objetivos de creación y absorción social de conocimiento están ausentes. El tinte distintivo de la concepción política que guía las actividades de fomento de las exportaciones en Argentina consiste en que, en general, esas actividades procuran el desarrollo productivo local mediante la derrama generada por las operaciones de comercio exterior, lo que se ve agravado por su discontinuidad en el tiempo.

Como dijimos anteriormente, lo que le da carácter de política al conjunto de actividades realizadas es la concepción económica que las orienta. Es decir, la forma como se concibe el proceso de desarrollo al que se aspira. Desde ese punto de vista, en el nivel taxonómico más general se puede discriminar entre las que están guiadas por objetivos comercialistas o bien desarrollistas. ${ }^{11}$ Los primeros tienen por finalidad directa el logro de exportaciones de bienes producidos localmente, mientras que los desarrollistas usan las ventas al exterior como instrumento para el desarrollo económico local. 0 sea, estos últimos consideran que una operación de exportación de bienes producidos localmente no es por sí misma un instrumento de desarrollo; para que lo sea hace falta la realización de ciertas actividades complementarias (Dabat, 2004a).

Las políticas comercialistas procuran facilitar las ventas de las empresas locales en el exterior, ya sea que fomenten el comercio aun cuando se realice por única vez, o que sólo fomenten las operaciones que se van a repetir en el tiempo, generando vínculos estables con el exterior. Esas políticas pretenden hacer una contribución al desarrollo productivo local en las experiencias de CPPp, en caso de que las operaciones facilitadas se repitan reiteradamente, mediante los beneficios sociales y privados del comercio y su función anticíclica en épocas de crisis. ${ }^{12}$ Esa expec-

\footnotetext{
${ }^{11}$ Esta clasificación es una abstracción realizada para pensar las PM CE en forma genérica. Aunque los municipios estudiados no se encuentran dentro de una de esas categorías en forma pura -tienen elementos de ambas-, en algunos casos se observó una marcada tendencia hacia una u otra categoría. Por ejemplo, en O lavarría (1996-2005) resulta clara la expectativa de que la derrama económica de los exportadores incida en el desarrollo productivo local, y en Junín (1996-2003) la preocupación estuvo centrada en el cómo generar desarrollo endógeno. Las entrevistas con los funcionarios y ex funcionarios municipales fueron aplicadas en los ocho municipios enumerados en la nota 3.

${ }^{12} \mathrm{Si}$ bien el mercado internacional no está exento de crisis recurrentes, éstas no han sido históricamente tan pronunciadas como las atravesadas por el mercado interno argentino, por lo que las oscilaciones en la demanda externa y en los términos de intercambio del país no invalidan la capacidad de las exportaciones como instrumento anticíclico en tiempos de crisis interna.
} 
tativa se apoya en la idea de que la ocupación permanente de un mercado externo es necesaria para explotar sus potencialidades anticíclicas porque la apertura de mercados externos suele ser muy lenta en relación con las urgencias que generan las crisis económicas. En esa lógica, esos espacios comerciales deberían mantenerse ocupados aun en épocas de auge para que estén disponibles en los momentos difíciles. No obstante, el requisito de continuidad en el largo plazo es especialmente difícil de cumplir en las cppp debido a su inestabilidad macro y microeconómica (Dabat, 2004a).

Entonces, por un lado existe una tendencia a la inestabilidad de las condiciones de producción en Argentina y, por otro, según lo demuestran los autores vinculados con la teoría del DLE, el desarrollo es un proceso de largo plazo en el que el afianzamiento de las relaciones de confianza entre los productores, el gobierno y otras instituciones crea un ambiente adecuado para la multiplicación de sinergias. Por lo tanto, cabe la pregunta: ¿ese enfoque endogenista es aplicable a cppp? Uno de los problemas más importantes de la aplicación de esa teoría a las localidades que nos ocupan es que en los países periféricos generalmente no hay estabilidad macroeconómica. Entonces los planes de largo plazo de aprendizaje productivo y acumulación tecnológica generalmente no tienen un contexto favorable, por lo que el papel anticíclico de las exportaciones podría ser más importante que en los países centrales.

Es decir, la aspiración desarrollista apunta que las exportaciones de una localidad argentina podrían cumplir una función anticíclica para dar estabilidad al cluster productivo en el que se apoya el proceso de construcción del desarrollo productivo local. Si su inserción en el mercado externo coadyuvara a que las empresas articuladas para generar sinergias tengan continuidad en su proceso de aprendizaje y de consolidación de las relaciones de confianza intra red, reduciría los efectos destructivos de las profundas crisis características de la los países periféricos (D abat, 2004a).

\section{Influencia esperada de las PM CE sobre el valor de los activos locales}

Según la tesis sostenida aquí, las exportaciones de bienes diferenciados realizadas en el marco de procesos de desarrollo de cPpP podrían coadyuvar a aumentar la capacidad de esos proce- 
sos para mejorar las condiciones en las que las empresas locales construyen su competitividad internacional y construir en el mediano y largo plazos una competitividad sistémica local que garantice estabilidad al proceso de desarrollo. Es decir, tienen capacidad potencial para ello. Podrían colaborar, entonces, con la formación de un círculo virtuoso para el desarrollo local en el que éste aumentara las exportaciones locales, lo que a su vez mejora las condiciones para un desarrollo local mayor, procurando el carácter reproductivo de su sistema articulado. Ese proceso podría recibir un aporte favorable de las PM CE a la creación y difusión social de tecnologías específicas para el aparato productivo local.

Ese círculo virtuoso lleva implícita la idea de valoración estratégica -véase nota 6- de ciertos activos disponibles para el desarrollo. Tal como se explicó al analizarse las experiencias exitosas de desarrollo local en países centrales, ese éxito estuvo basado en una conjunción de valoración de activos sociales y privados disponibles para el desarrollo y el uso de esos activos con fines de desarrollo. La combinación de valoración de activos con el uso de los propios activos valorados es condición necesaria en cualquier estrategia de desarrollo productivo endógeno realizada en una cPpp.

Por lo tanto, y tal como fue planteado en el punto anterior (2), la creación del círculo virtuoso comercio internacional-desarrollo local endógeno no es, ni puede ser, automática. N ecesariamente es un proceso de largo plazo. Entonces, teniendo en cuenta la inestabilidad del mercado en Argentina, para que el comercio genere condiciones favorables para el desarrollo local debe ser orientado por políticas explícitas sostenidas en el largo plazo. Éstas se deberían encargar de facilitar la difusión de los beneficios de la internacionalización de empresas locales en la estructura económica del lugar. Así, las exportaciones de bienes producidos localmente podrían operar sobre la acumulación y valoración estratégica de activos sociales y privados radicados allí.

Esos activos pueden agruparse entre estáticos o de baja capacidad de reproducción endógena y dinámicos o de alta capacidad de reproducción endógena. Ambos tipos de activos podrían ser sometidos a procesos de valoración vinculados con el comercio internacional, entre otros factores. Además de la valoración que circunstancialmente ocasiona en los activos el comercio internacional, éste crea condiciones favorables para el aprendizaje productivo y comercial de las empresas exportadoras y de sus proveedores, lo 
que podría repercutir favorablemente sobre las condiciones de reproducción endógena de los activos dinámicos. Esta idea podría sintetizarse en que en Argentina la exportación de producción local tiene capacidad potencial como mecanismo de valoración de activos estáticos, por medio de la ampliación del mercado, con la consecuente explotación de economías de escala, y de activos dinámicos, por medio del establecimiento de condiciones para el aprendizaje y el desarrollo de capacidad innovadora.

\section{El proceso de valoración estratégica de los activos disponibles para el desarrollo}

Para explicar las diferencias que existen en el significado del comercio exterior en los procesos de desarrollo local en los distintos tipos de ciudades (CPPP y CPPC) fue adoptado el supuesto de que en el entorno en el que se sitúan las cppp hay proporcionalmente más activos estáticos y en el de las CPPC se invierte la relación, lo que permite ensayar algunas reflexiones sobre la insuficiencia que la teoría del DLE tiene para ser aplicada a cPPP. De ese supuesto se desprende que si el proceso de desarrollo, y consecuentemente de valoración, de los activos dinámicos marcha exitosamente, la proporción de dotación de activos debería irse revirtiendo con el transcurso del tiempo gracias al carácter autorreproductivo de los activos dinámicos.

Los activos estáticos tienen carácter exógeno; es decir, no se reproducen a sí mismos. Éstos no necesariamente son utilizados para la producción eficiente y para el desarrollo. Entre los componentes de esta categoría de activos se encuentran: los recursos naturales, que incluyen factores como la fertilidad del suelo, el clima, la riqueza minera, etc.; los recursos materiales (capital), que abarcan capacidad industrial instalada, presencia de grandes empresas, producción de commodities y bienes no transables, capacidad de inversión de los empresarios de la región, etc., cuando sus articulaciones locales no desatan potencialidades autorreproductivas; la dotación de mano de obra calificada pero no involucrada con el agrupamiento dinámico del desarrollo y la no calificada, el tamaño de estado municipal y el del mercado interno en relación con la escala óptima de producción, y la ubicación geográfica del municipio.

Esos activos pueden ser valorados por la exportación de bienes intensivos en cada uno de ellos pero difícilmente dinamizarán las relaciones económicas y tecnológicas locales. N o obstan- 
te, ciertos activos estáticos pueden cumplir una función estratégica clave en aquellos lugares donde la economía local gira en torno a ellos, especialmente en los pasos iniciales del proceso de desarrollo. A medida que el aprendizaje y el contacto con un mundo más dinámico alteran la conducta de las empresas arraigadas en el territorio, es esperable que aumente la reproducción de los activos dinámicos y, por lo tanto, que tengan una importancia estratégica relativamente menor los activos estáticos. ${ }^{13}$

Los activos dinámicos, en cambio, tienen carácter endógeno porque se reproducen a sí mismos. Entre los componentes de esa categoría de activos se encuentran la inversión endógena, la marca ciudad, las habilidades de la mano de obra calificada localmente disponible, el conocimiento productivo y organizacional de la firma, y el conocimiento acumulado en el sistema productivo o agrupamiento que contiene a las firmas exportadoras.

La inversión endógena es aquella que se produce en la localidad como consecuencia del ahorro generado en el propio lugar y se reinvierte en él. Es resultado del aumento de la productividad y la eficiencia que genera el desarrollo productivo local y, a su vez, es fuente de mayor desarrollo. En eso consiste su carácter endógeno. Por lo tanto, incide sobre la capacidad exportadora local en forma dinámica, a diferencia de lo que ocurre con la inversión cuya utilidad no es reinvertida en el proceso productivo local. El comercio exterior de la producción local, por su parte, mejora las posibilidades de reproducción endógena del capital al cumplir una función anticíclica que permite la estabilidad que requiere el agrupamiento dinámico del desarrollo local.

La marca ciudad es un activo intangible social, basado en la calidad de la producción garantizada en forma comunitaria y valorado por la publicidad ante los mercados potenciales de los productos locales. Esa marca generalmente se orienta y desarrolla hacia un sector 0 segmento de sector productivo 0 a bienes con características muy acotadas, donde la localidad que la desarrolla tiene ventajas competitivas. Ese activo puede ser de fundamental importancia en el tipo de localidades que nos ocupa porque, dado

\footnotetext{
${ }^{13} \mathrm{H}$ ay por lo menos tres corrientes interpretativas que explican la relación entre la exportación de bienes de una pyme y su evolución: el enfoque de internacionalización por aprendizaje -enfoque de Uppsala- (J ohanson y Wiedersheim-Paul, 1975; J ohanson y Vahlne, 1977 y 1990), el de innovación (Lee y Brasch, 1978; Cavusgil, 1998) y el de redes (Brown y Cook, 1990; Johanson y Vahlne, 1990; Ellis, 2000). Los tres comparten el criterio de que a mayores destrezas de la firma y/o de los empresarios que la dirigen, mejores son las condiciones que las empresas tienen de internacionalizarse y de aprovechar las oportunidades que brinda el mercado internacional para evolucionar en su funcionamiento.
} 
que las pymes que no tienen larga historia en el comercio internacional todavía no tienen prestigio propio, la marca ciudad da una referencia sobre la calidad y la seriedad del proyecto. Las exportaciones sostenidas en el tiempo hacia los mismos mercados ayudan a consolidar la marca ciudad, dado que demuestran la calidad de la producción local. Por lo tanto, alimentan el mecanismo de revaloración endógeno de ese activo.

0 tro activo de fundamental importancia es de los conocimientos productivos individuales de trabajad ores cal ificad os local mente disponibles. Se trata de conocimientos de los trabajadores de las empresas e instituciones de apoyo o que están desocupados en la ciudad y son reproducibles endógenamente. En principio, la mano de obra calificada no ocupada en el núcleo endógeno del desarrollo es un activo potencial que se convertiría en activo efectivo al ser contratada por una firma perteneciente al núcleo. La exportación de bienes producidos localmente puede incidir en forma directa en el aprendizaje de los trabajadores de la firma exportadora, y en forma indirecta sobre los conocimientos de los trabajadores de las empresas proveedoras y demás instituciones vinculadas con el agrupamiento.

El conocimiento colectivo de la firma ayuda a elevar la productividad del trabajo y a crear habilidades específicas de la firma que superan a la suma del conocimiento individual de sus trabajadores, lo que mejora las condiciones para la inversión en capacitación y para realizar esfuerzos por mejorar constantemente la organización del trabajo colectivo. Así se genera el círculo virtuoso que le da al conocimiento colectivo el carácter de activo dinámico endógeno. El requisito de su capacidad de autorreproducción pone la atención en la estrategia tecnológica de las firmas y de las redes locales. La adquisición de paquetes tecnológicos abiertos, aun cuando sean más caros que los cerrados, generalmente mejora las condiciones para la evolución de las habilidades de la mano de obra calificada local. Suele implicar un proceso menos rentable en el corto plazo que el basado en tecnologías cerradas pero de eficiencia y eficacia crecientes, por lo que puede ser mucho más aconsejable para proyectos de desarrollo de largo plazo. La relación de causalidad exportación-valoración del activo tiene, entonces, aspectos vinculados con la relación paquetes tecnológicos-exportación, por lo que el carácter endógeno de ese activo depende de la dependencia formal o tácita que las firmas exportadoras y las relacionadas con ellas tengan con sus proveedores tecnológicos. 
El activo basado en el conocimiento colectivo del sistema local Ileva a la competitividad de las firmas que lo componen a depender de la lógica de red. Por lo tanto, la homogeneidad de las tecnologías y prácticas utilizadas por las distintas firmas pertenecientes a ese agrupamiento productivo pasa a cumplir un papel preponderante para el desarrollo local. Dicha homogeneidad ayuda a articular el aparato productivo local reduciendo el costo de transacción y compatibilizando el sendero tecnológico de largo plazo de las firmas pertenecientes al agrupamiento. Esa articulación es una exigencia de la competencia internacional que requiere mucho más que la eficiencia dentro de la empresa que exporta. Para poder competir internacionalmente en mercados dinámicos de productos diferenciados hace falta un sistema competitivo. Pero, en la medida en la que para acceder a mercados externos las firmas locales deben adaptarse a pautas tecnológicas heterogéneas entre sí, disminuyen las posibilidades de constituir un cluster exportador con dinámica propia de reproducción y aprendizaje. Por lo tanto, las exportaciones de bienes locales diferenciados pueden ser un motor para la articulación del aparato productivo interno 0 un freno. Esto es, depende de la estrategia de desarrollo local seguida por los actores intervinientes.

Por último cabe aclarar que todo esto no pretende quitarle méritos a la mencionada teoría, que es el avance teórico más importante para analizar problemas de desarrollo local desde una perspectiva económica. Por el contrario, es un intento de examinar problemas todavía no explorados desde esa concepción para ayudar a su consolidación y capacidad explicativa.

\section{Conclusiones}

Durante la década la pasada, un número considerable de gobiernos municipales argentinos comenzó a realizar actividades de fomento de exportaciones locales. Esas actividades se realizan en el marco de políticas implícitas orientadas a fortalecer las capacidades endógenas del territorio para el desarrollo productivo local. El sentido político de ese conjunto de actividades tiene dos orígenes teóricos: la economía de mercado y el desarrollo local endógeno. Ambos son insuficientes para incidir favorablemente sobre procesos locales de desarrollo. El primero resulta ingenuo por las limitaciones que tiene el mercado para seleccionar eficazmente las trayectorias evolutivas más idóneas para el desa- 
rrollo, lo que se agrava por la inestabilidad macroeconómica recurrente en Argentina. El segundo, si bien está más comprometido con la acumulación de capacidades locales para el desarrollo, está inspirado en el éxito que tuvieron diversas experiencias europeas, donde el contexto es muy diferente al argentino.

La experiencia internacional muestra que la exportación de bienes diferenciados producidos en el territorio es una condición útil para el DLE pero no suficiente, ni siquiera necesaria. Por lo tanto, las PM CE pueden servir para el desarrollo de CPPP, aunque no pueden garantizarlo. En muchas experiencias exitosas de desarrollo local realizadas en Europa queda demostrada la importancia de: a) las capacidades del territorio para la competitividad internacional y de la inserción internacional para fortalecer las capacidades del territorio; b) el sistema productivo local con la activa participación de sus pymes debido al achicamiento del tamaño medio de las plantas y a la necesidad de contar con capacidad de flexibilidad para la adaptación a las circunstancias cambiantes del entorno; c) la necesidad de contar con ciertas habilidades en las firmas para aprender en el mercado internacional de las técnicas más avanzadas y la creación de condiciones favorables para crear esas habilidades cuando su presencia en el mercado internacional es permanente en el largo plazo; d) los activos sociales y privados disponibles para el desarrollo que tienen mayor capacidad de autorreproducción y valoración del territorio, y e) la exportación de bienes diferenciados es un instrumento fundamental para valorar los activos sociales disponibles para el desarrollo construido a partir del uso social eficiente de esos activos.

N o obstante, la experiencia europea no es perfectamente replicable en Argentina.

La imperfecta replicabilidad de aquellas experiencias se debe a la condición de territorios periféricos de los municipios argentinos. Tiene dos implicancias básicas sobre la capacidad potencial de sus políticas de comercio exterior para fortalecer procesos de desarrollo endógeno:

- La mayor proporción relativa de activos estáticos en los territorios periféricos que en los centrales complejiza la capacidad de autorreproducción y valoración sistémica, por lo que la función que las exportaciones fomentadas por los gobiernos municipales podría cumplir en el desarrollo lo- 
cal de las cppp es diferente a la que cumplen las exportaciones locales en los países centrales.

- El mecanismo por el que el comercio exterior puede incidir sobre los procesos de DLE se basa en la valoración estratégica de activos disponibles en los procesos de construcción social del desarrollo, lo que no puede ocurrir en forma automática ya que el desarrollo es un proceso de construcción social de largo plazo. Para que eso ocurra hace falta que el Estado y demás actores del proceso promuevan la difusión social de los beneficios del comercio, encuadrándolos en una estrategia de desarrollo.

Entonces, uno de los aportes que puede hacer el Estado municipal para el desarrollo local en Argentina es la aplicación de PM CE pensadas desde adentro del territorio para articular los activos disponibles y valorarlos en el mercado internacional, compensando la inestabilidad estructural que desarticula permanentemente las cadenas de valor endógenas y conspira contra el aprendizaje colectivo de todos sus eslabones, y la escasa competitividad del aparato productivo local en relación con la de los situados en los países centrales.

De todo esto se desprende que la exportación de bienes diferenciados en los procesos de desarrollo de ciertas cPpP podría ser eficaz, a la vez que el desarrollo productivo local mejora las condiciones para que las empresas locales eleven su competitividad en términos internacionales. Por lo tanto, el desarrollo local puede aumentar las exportaciones locales, lo que a su vez mejoraría las condiciones para un desarrollo local mayor, generando un círculo virtuoso que cree condiciones favorables para el desarrollo. Círculo que podría comenzar a crearse por las políticas municipales de competitividad y comercio exterior. N o obstante, las políticas configuradas por medio de las actividades de fomento de exportaciones realizadas hasta el momento, en general, no están orientadas a propiciar condiciones de largo plazo favorables para la valoración de los activos disponibles para el desarrollo sino a resolver problemas puntuales de los empresarios locales.

Por lo tanto, la evidencia empírica disponible sobre los procesos desarrollados hasta ahora en Argentina no despierta mayor optimismo dado que se manifiesta un enfoque erróneo sobre las características que deberían asumir los procesos de desarrollo de las cppp. De la correcta teorización sobre la potencialidad que 
ofrece el crecimiento del valor estratégico para el desarrollo de cada tipo de activo respecto a la exportación de los bienes producidos localmente depende la comprensión de las oportunidades que encierran las PMCE para el desarrollo de cPpP. 


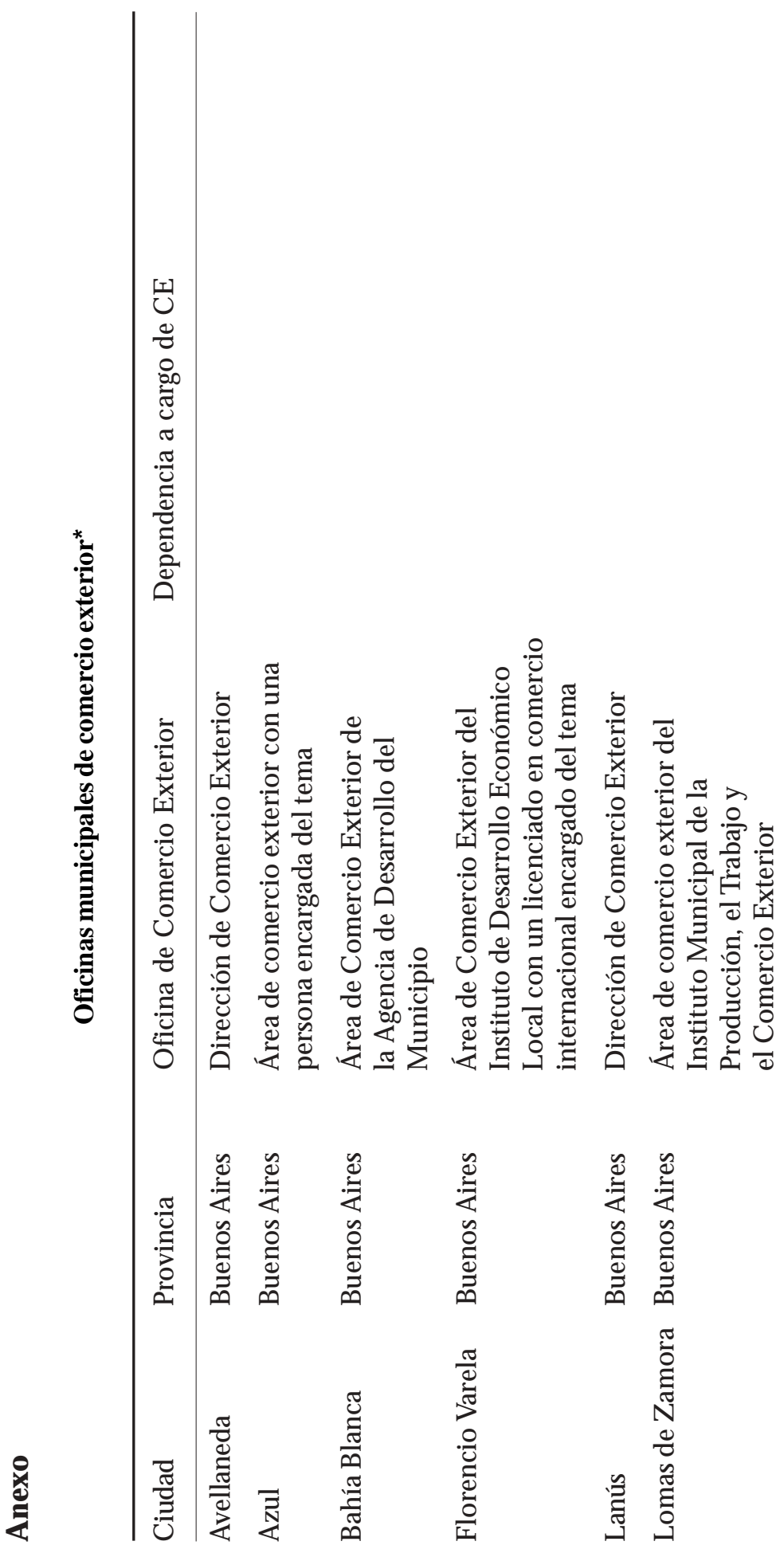




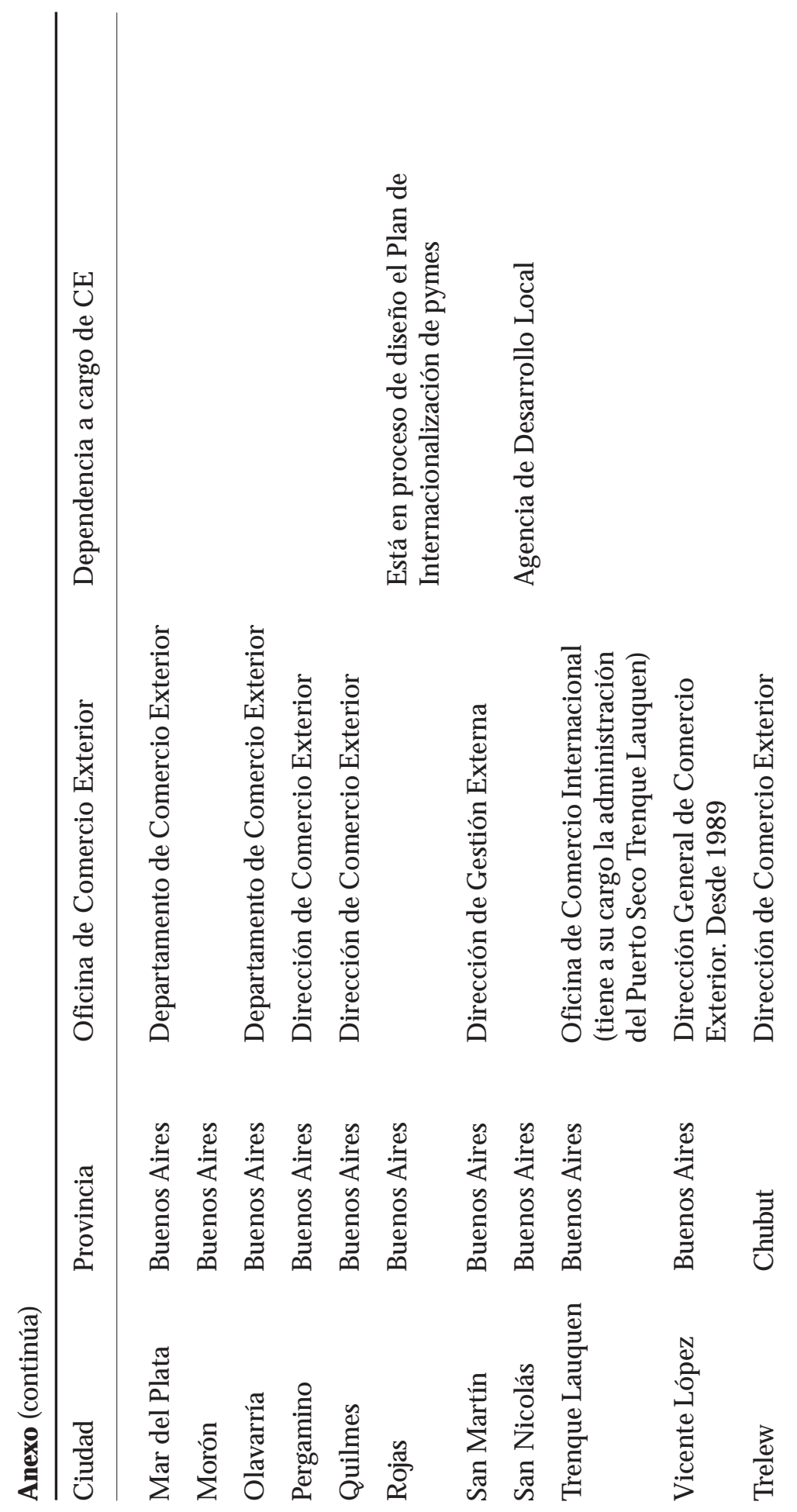




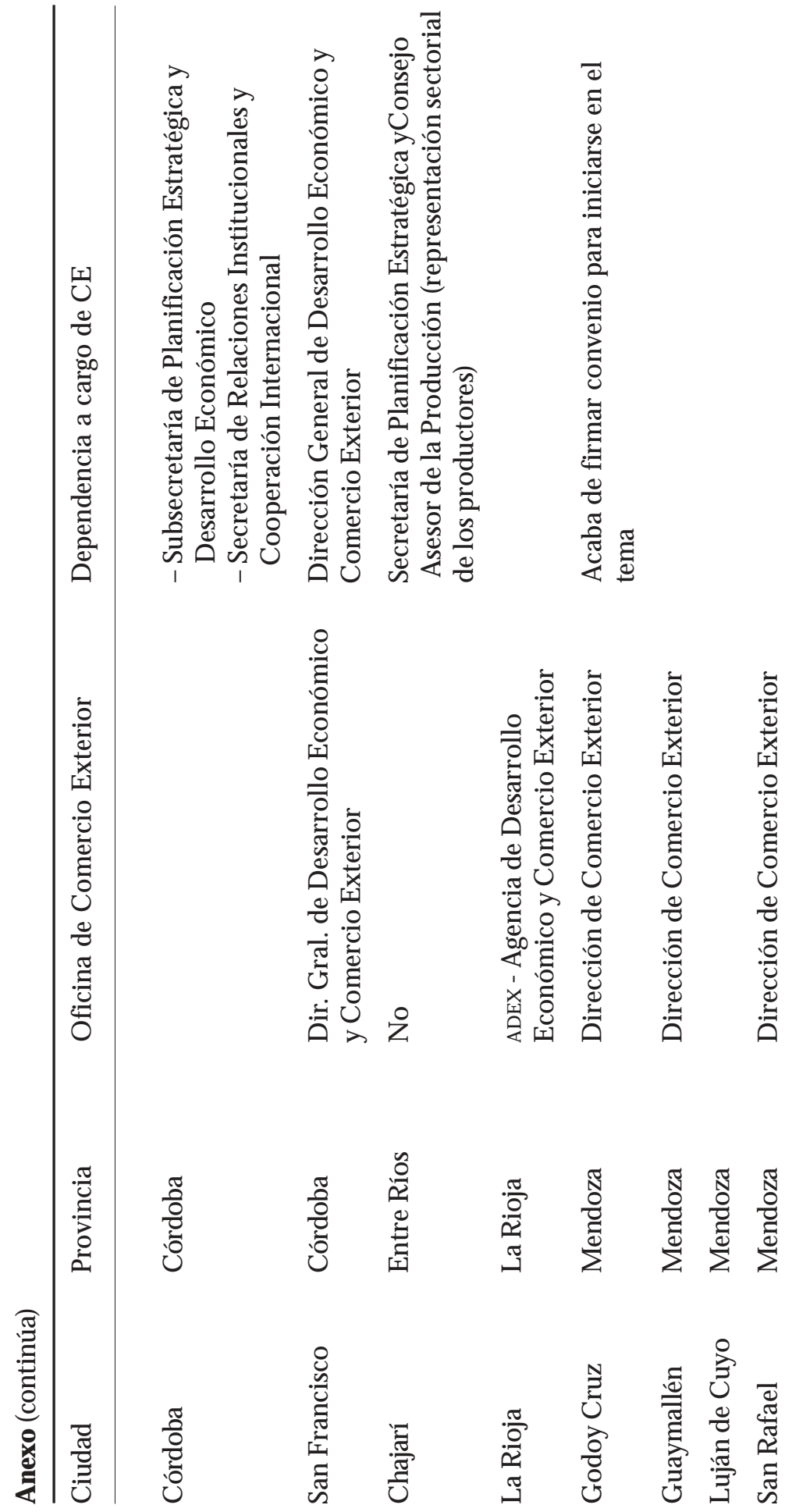




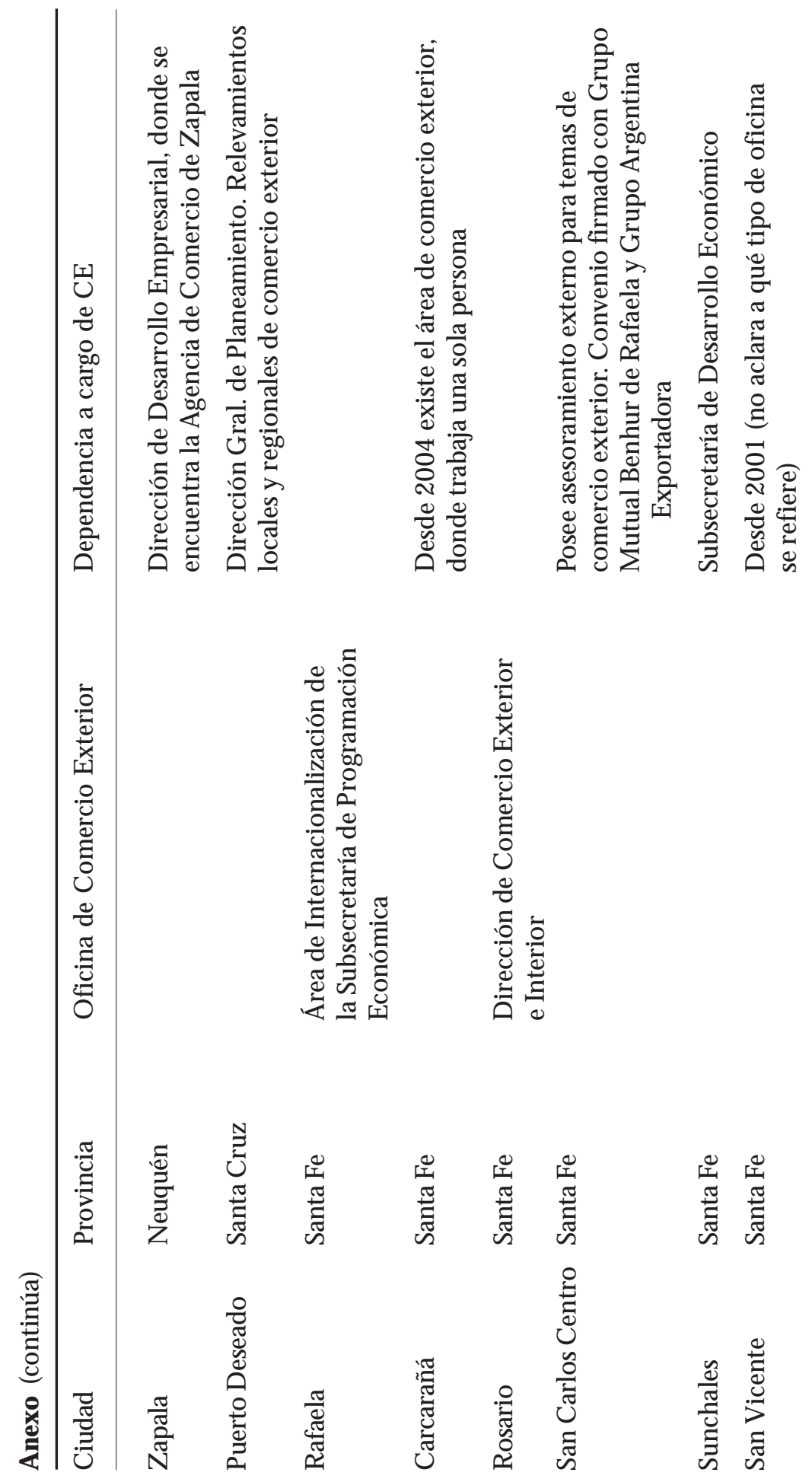




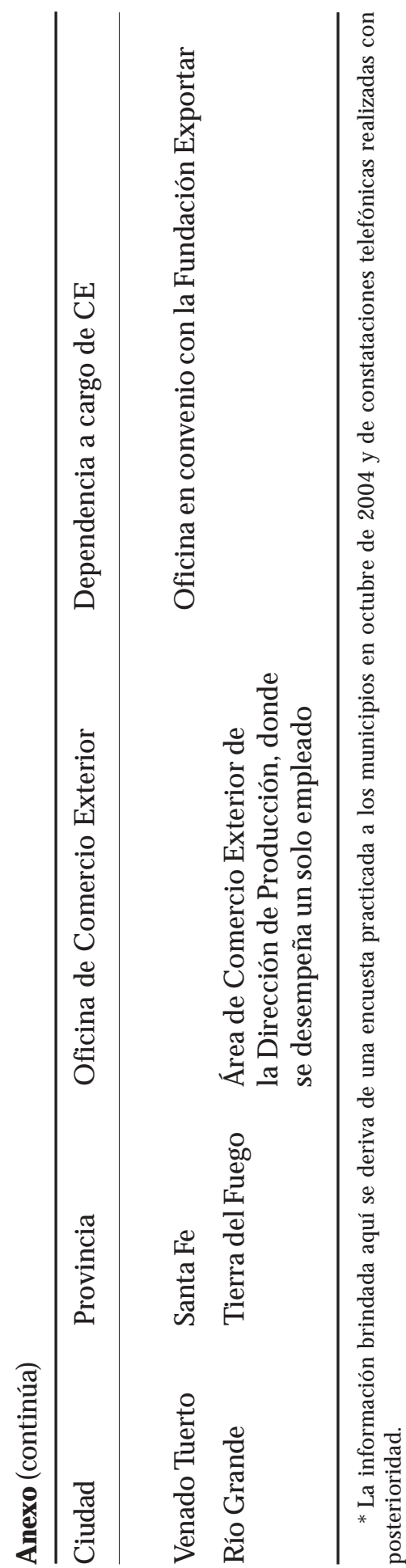




\section{Bibliografía}

Alburquerque, Francisco (1995), Competitividad internacional, estrategia empresarial y papel de las regiones, D ocumento 95/28, Comisión Económica para América Latina, Serie Ensayos, Santiago de Chile.

(1996), Desarrollo económico local y distribución del progreso técnico. Una respuesta a las exigencias del ajuste estructural, ILPES/C epal, Santiago de Chile.

(1997), M etodología para el desarrollo económico local, ILPES/C epal, Santiago de Chile.

(2001), "La importancia del enfoque del desarrollo económico local", en Antonio Vázquez Barquero y Ó scar M adoery (comps.), Transformaciones globales, instituciones y políticas de desarrollo local, H omo Sapiens Ediciones, Rosario, Argentina.

(2004), El enfoque del Desarrollo Económico Local, Organización Internacional del Trabajo, Cuadernos de Desarrollo Local núm. 1, Buenos Aires.

Becattini, Giacomo y Enzo Rullani (1996), "Sistemas productivos locales y mercado global", Información Comercial Española. Revista de Economía, M adrid, junio, 754: 1124.

Bianchi, Patrizio (1997), Construir el mercado, Universidad Nacional de Q uilmes Ediciones, Buenos Aires.

Brown, Rick y David Cook (1990), "Strategy and Performance in British Exporters", Quarterly Review of Marketing, primavera, 15 (3): 42-61.

Burgueño, O. y L. Pitaluga (1994), “El enfoque neo-schumpeteriano de la tecnología", R evista Q uantum, M ontevideo 1 (3).

Castells, M anuel (2001), La galaxia Internet, Plaza \& Janés, Madrid. 
Cavusgil, S. Tamer (1998), "Perspectives: Knowledge Development in International M arketing", Journal of International Marketing, 6 (2): 103-112.

Dabat Germán (2003), "Políticas municipales de competitividad y comercio exterior", en Germán Dabat (coord.), D esde adentro. Políticas municipales de competitividad y comercio exterior, Universidad Nacional de Quilmes Ediciones, Quilmes, pp. 21-54.

(2004), conferencia dictada en el seminario La G lobalización y su Impacto en el Desarrollo Local, Universidad $\mathrm{N}$ acional del N oroeste de la Provincia de Buenos Aires, Lincoln, 6 de agosto.

y N éstor Le Clech (2004), "La política de comercio exterior del municipio de Florencio Varela", Argirópolis, Universidad $\mathrm{N}$ acional de $\mathrm{Q}$ uilmes.

(2004b) "Las políticas municipales de competitividad y comercio exterior en Argentina. La perspectiva de la teoría del desarrollo económico", Argirópolis, Universidad $\mathrm{N}$ acional de La Plata, Universidad N acional del Litoral y Universidad N acional de Quilmes, Argentina.

(2005) Las políticas municipales de competitividad y comercio exterior en Argentina como instrumento del desarrollo local endógeno, borrador de tesis doctoral, Universidad Autónoma de Zacatecas, M éxico.

Dosi, Giovanni, Keith Pavitt y Luc Soete (1990), The Economics of Technical Change and International Trade, $\mathrm{H}$ arvester Wheatsheaf, Londres.

Ellis, Paul (2000), "Social Ties and Foreign M arket Entry", Journal of International Bussines Studies, 31 (3): 443-469.

Finquielevich, Susana (2001), "Ciudades en el espacio de las redes: $\mathrm{N}$ uevas centralidades y periferias urbanas en la sociedad informacional", Mundo Urbano, revista virtual, Universidad Nacional de Quilmes, Argentina, http:// 
www.argiropolis.com.ar/mundourbano/anteriores/14/ Editorial.htm, octubre de 2004.

J ohanson, Jan y Finn Wiedersheim-Paul (1975), "The Internationalization of de the Firm-four Swedish Cases", Journal of Managment Studies, octubre, 12: 305-322.

Johanson, Jan y Jan-Erik Valhne (1977), “The Internationalization Process of the Firm: A M odel of Knowledge Development and Increasing Foreign $M$ arket Commitments", Journal of International Business Studies, 8 (1): 23-32.

(1990), "The M echanism of Internationalization", International Marketing Review, 7 (4): 11-24.

Katz, Jorge (1976), Importación de tecnología, aprendizaje e industrialización dependiente, Fondo de Cultura Económica, M éxico.

Kosacoff, Bernardo y Andrés López (1998), "Cambios tecnológicos y organizacionales en las pymes", en Ricardo Kesselman (coord.), Las pymes. Clave del crecimiento con equidad, Grupo Socma, Buenos Aires, pp. 121-154.

Krugman, Paul (1992), G eografía y comercio, Antoni Bosch, Barcelona.

Leal, M arina (2004), “Experiencias de desarrollo económico local en el centro de la provincia de Buenos Aires: Olavarría y Rauch", Argirópolis, UNLP-UNL-UNQ, http:// www.argiropolis.com.ar, octubre de 2004.

Lee, Woo-Young y John Brasch (1978), "The Adoption of Export as an Innovative Strategy", Journal of International Business Strategy, 9 (1): 85-93.

Porter, M ichael (1991), Las ventajas competitivas de las naciones, Javier Vergara, Buenos Aires.

Saba, Andrea (1997), El model o italiano. La especialización flexibley los distritos industriales, Universidad N acional de La Plata, La Plata, Argentina. 
Segreto, Vanina (2004), "PM CE. Casos Lomas de Zamora y Q uilmes", Argirópolis, UnLP-UNL-UNQ, http://www.argiropolis. com.ar, octubre de 2004.

Silva Lira, I ván (2003), M etodol ogía para la elaboración de estrategias de desarrollo local, Instituto Latinoamericano y del Caribe de Planificación Económica y Social (ILPES), Serie Gestión Pública núm. 42, Santiago de Chile.

Varian, H al R. (1998), Microeconomía intermedia, 4a ed., Editorial Antoni Bosch, Barcelona.

Vázquez Barquero, Antonio (1993), Política económica local, Pirámide, M adrid.

(1999), Desarrollo, redes e innovación. Lecciones sobre desarrollo endógeno, Pirámide, M adrid.

(2005), Las nuevas fuerzas del desarrollo, Antoni Bosch, Barcelona.

Villar, Alejandro (2004), "Una década de desarrollo local en Argentina. Balance y perspectivas", M undo Urbano, revista virtual, Universidad N acional de Q uilmes, Argentina, 24, http://www. mundourbano.unq.edu.or/index.php? option $=$ com_content $\&$ task $=$ view $\&$ id $=172 \&$ Itemid $=50$.

Recibido: 12 de abril de 2005. Reenviado: 2 de febrero de 2006. Aceptado: 9 de marzo de 2006.

Germán Dabat es licenciado en economía por la Universidad $\mathrm{N}$ acional Autónoma de $\mathrm{M}$ éxico, master en desarrollo económico en América Latina por la Universidad Internacional de Andalucía, y está por culminar el doctorado en estudios del desarrollo de la Universidad Autónoma de Zacatecas, con el proyecto de tesis doctoral Políticas municipales de comercio exterior en la Argentina. Perspectiva de la teoría del desarrollo. Fue becario de varias instituciones académicas de M éxico, Argentina y España. Actualmente es profesor de las asignaturas M odelos de Desarrollo Económicos Comparados y Política Económica y Comercial Argentina en la Licenciatura en Comercio Internacional de la Uni- 
versidad $\mathrm{N}$ acional de Quilmes, y Desarrollo Económico en la $M$ aestría en Políticas de M igración Internacional de la Universidad de Buenos Aires. Fue consultor externo de la O rganización de $\mathrm{N}$ aciones Unidas (Buenos Aires). De sus publicaciones sobresaIen: Desde adentro. Políticas municipales de competitividad y comercio exterior, Editorial Universidad Nacional de Quilmes, 2003, de la que fue coordinador y autor de los artículos "Políticas municipales de competitividad y comercio exterior" y "Tecnología para el desarrollo de la competitividad local en el mercado internacional"; también se encuentra: "Tecnologías en las políticas municipales de comercio exterior", DHIAL, publicación electrónica del Instituto Internacional de Gobernabilidad (www.iigov.org/dhial), Barcelona, mayo de 2002; "Políticas locales de comercio exterior en la Argentina: comercio y desarrollo desde una perspectiva endogenista", Revista Comercio Exterior, Banco N acional de Comercio Exterior, M éxico, mayo de 2005; y con N . Le Clech, "La política de comercio exterior del municipio de Florencio Varela", Argirópolis, Universidad Nacional de Quilmes, marzo de 2004. Ha participado como panelista, comentarista y ponente en 17 actos académicos. 